Y.-H. Chu, N.B. Suntzeff, J.E. Hesser, and D.A. Bohlender, eds.

\title{
Massive Star Evolution
}

\author{
N. Langer \\ Institut für Physik, Universität Potsdam \\ Postfach 601553, D-14415 Potsdam, Germany
}

\author{
A. Heger ${ }^{1}$ \\ Max-Planck-Institut für Astrophysik \\ Postfach 1523, D-85740 Garching, Germany
}

\begin{abstract}
The evolution of massive stars is far from being fully understood, as we outline by pointing to a number of open problems related to massive stars in the Magellanic Clouds. We argue that rotation may be a key ingredient in the physics of massive stars. We report on recent results obtained including rotation, and their relevance to these remaining questions.
\end{abstract}

\section{Introduction: Current Problems in Massive Star Evolution}

The quantitative theory of stellar structure is more than 100 years old (Emden 1907). Our understanding of the stellar interior has improved dramatically since then, especially so since the 1950 's when it became possible to construct detailed stellar models with the help of computers. Although there are still many fundamental questions left in the field of low- and intermediate mass stars, we can say that their evolutionary path - i.e., their route from the main sequence through the red giant and asymptotic giant branch to the white dwarf stage - has been known in detail for several decades (see, for example, Iben 1974). In addition, the principles of the evolution of massive stars' cores through the major nuclear fusion stages from hydrogen to silicon burning have also been known for a similar length of time (Weaver et al. 1978).

However, even today our understanding of the evolution of the observable properties of massive stars $\left(M_{\mathrm{ZAMS}} \gtrsim 8 \mathrm{M}_{\odot}, \log L / \mathrm{L}_{\odot} \gtrsim 4\right)$ is still rudimentary, as demonstrated by the following six problems related to massive stars in the Magellanic Clouds, a list which is far from complete.

1. Where is the post-main sequence gap in the distribution of massive stars in the HR diagram of the LMC (Blaha \& Humphreys 1989; Fitzpatrick \& Garmany 1990)? It is predicted by all stellar evolution calculations (Fig. 9 in Langer 1991) but there is no trace of it in the data. Can this gap be filled by secondary stars in massive close binary systems (Fig.1)?

\footnotetext{
${ }^{1}$ present address: UCO/Lick Observatory, UC Santa Cruz, CA 95064, USA
} 


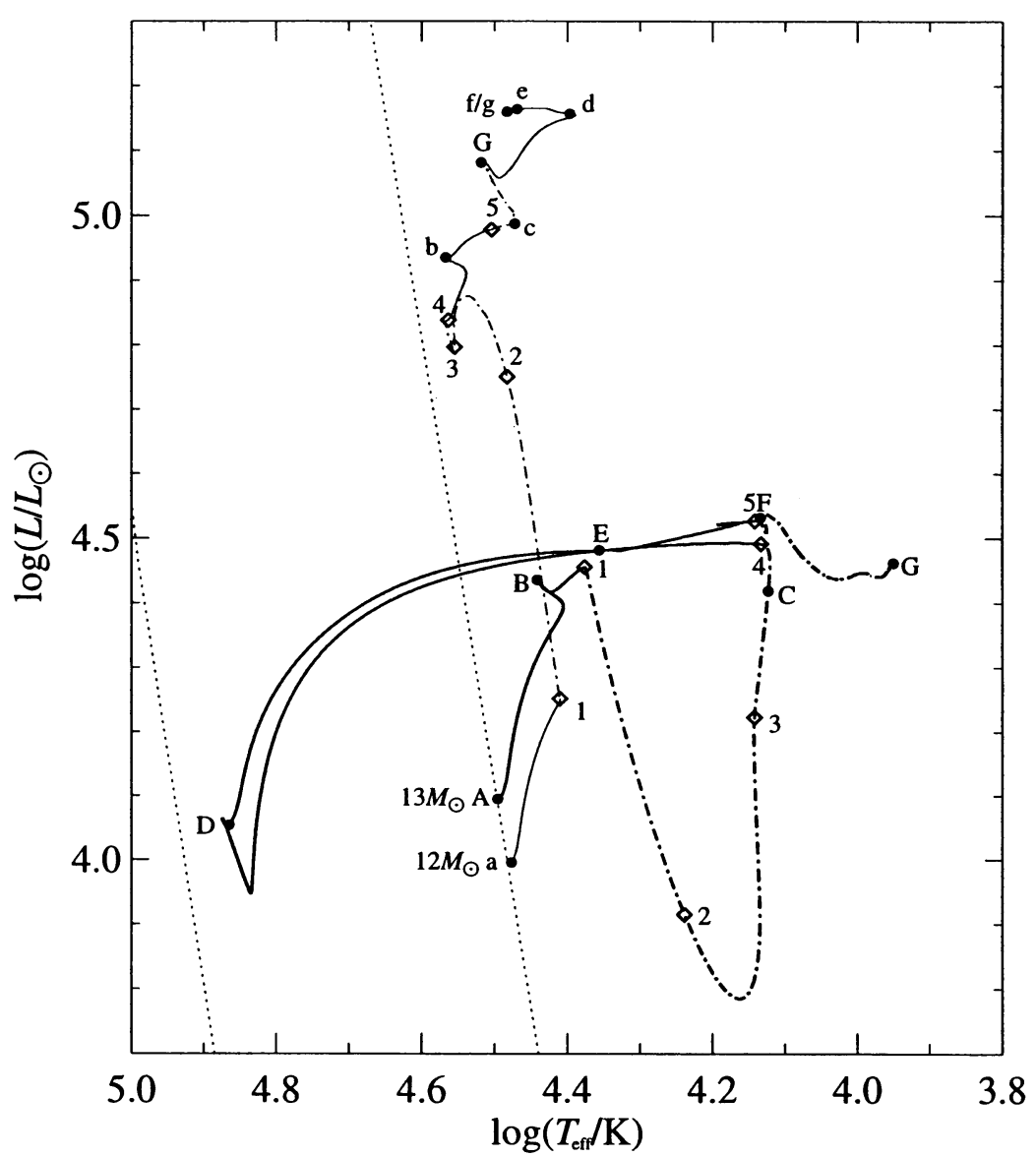

Figure 1. Evolutionary tracks in the HR diagram of the components of a $13+12 \mathrm{M}_{\odot}$ case B close binary system with a metallicity of $Z_{\odot} / 4$ and an initial period of 3.1 days. The path of the primary component (initial mass $13 \mathrm{M}_{\odot}$ ) is marked by the thick line and upper case letters, that of the secondary by the thin line and lower case letters. Mass transfer stages correspond to the dot-dashed parts of the lines. The thin dotted lines designate the zero age main sequence and the location of pure helium stars (helium main sequence). The letters designate beginning and end of nuclear burning stages, i.e., core hydrogen burning $(\mathrm{a} / \mathrm{A}-\mathrm{b} / \mathrm{B})$, core helium burning $(\mathrm{c} / \mathrm{C}-\mathrm{d} / \mathrm{D})$, core carbon burning $(e / E-f / F)$, and neon ignition $(g / G)$. Numbers designate mass transfer events for both stars. 1: beginning of Case B mass transfer, 2: maximum of mass transfer rate, 3 : start of slow phase of Case B mass transfer, 4: end of Case B mass transfer, 5: start of Case BB mass transfer. The final masses of the primary and secondary are $1.6 \mathrm{M}_{\odot}$ and $22 \mathrm{M}_{\odot}$, respectively. Note that this kind of binary evolution may be relevant to problems 1) and 3) mentioned in Section 1. See Braun \& Langer (1999) for more details. 
2. The observed trend of the blue-to-red supergiant ratio as function of the metallicity is opposite to the one predicted by any stellar evolution models (Langer \& Maeder 1995).

3. When in 1987 a blue supergiant exploded as a supernova in the Large Magellanic Cloud (Arnett et al. 1989) it was a surprise to the community. The reason for the blue color of the progenitor star is still not unambiguously explained (see Langer et al. 1989, Woosley et al. 1998, and see Fig. 1).

4. What is the origin of the $\mathrm{B}[\mathrm{e}]$ supergiants primarily found in the Magellanic Clouds (Gummersbach et al. 1995), i.e., how can evolved supergiants have disks or disk winds around them (Langer \& Heger 1998)?

5. What is the evolutionary status of the WNL/Ofpe stars which again are primarily found in the Magellanic Clouds (Pasquali et al. 1997; Crowther \& Bohannan 1997)?

6. Does the upper stellar mass limit depend on metallicity? While extremely massive stars $\left(\sim 200 \mathrm{M}_{\odot}\right)$ are found in the LMC (Kudritzki et al. 1996), recent investigations of stars near the Galactic Center (Figer et al. 1998) indicate the presence of very massive stars also in high metallicity environments.

The uncertainty in massive star evolution is dominated by the lack of understanding of two kinds of processes: the physics of mass loss from the stellar surface, and the physics of internal mixing processes (Langer 1994). In the present paper, we want to outline that both processes, despite acting in quite different locations in massive stars, may be strongly influenced by a process which has mostly been neglected in past studies of massive star evolution: rotation.

\section{Massive Star Evolution with Rotation}

Massive stars are rapid rotators. Equatorial rotation velocities span the range $v_{\text {rot }}=100-400 \mathrm{~km} \mathrm{~s}^{-1}$, with B stars rotating closest to their break-up speed $v_{\text {crit }}$, and values of $v_{\text {rot }} / v_{\text {crit }}=\omega_{\text {rot }} / \omega_{\text {crit }}=0.5$ frequently observed for $\mathrm{O}$ stars (e.g., Howarth et al. 1997). During the last decade, many observations have revealed unusual surface abundances that may require additional internal mixing (beyond that of plain convection) for their explanation (Langer et al. 1998, and references therein).

Fig. 2 gives an overview on the evolution of some surface properties of massive star models which include mixing due to rotationally induced instabilities and the effect of the centrifugal force. For the most massive models with the fastest rotation, one can even obtain a transition to Wolf-Rayet (WNL) type abundances (see also Langer 1992), which may be relevant for understanding the WNL/Ofpe stars (Pasquali et al. 1997). Furthermore, for a distribution of initial rotation rates, one obtains, at a given initial mass, a spread in main sequence luminosities and effective temperatures (Fig. 3). This might reduce the problem of the missing post-main sequence gap mentioned above, but seems not to solve it completely. 


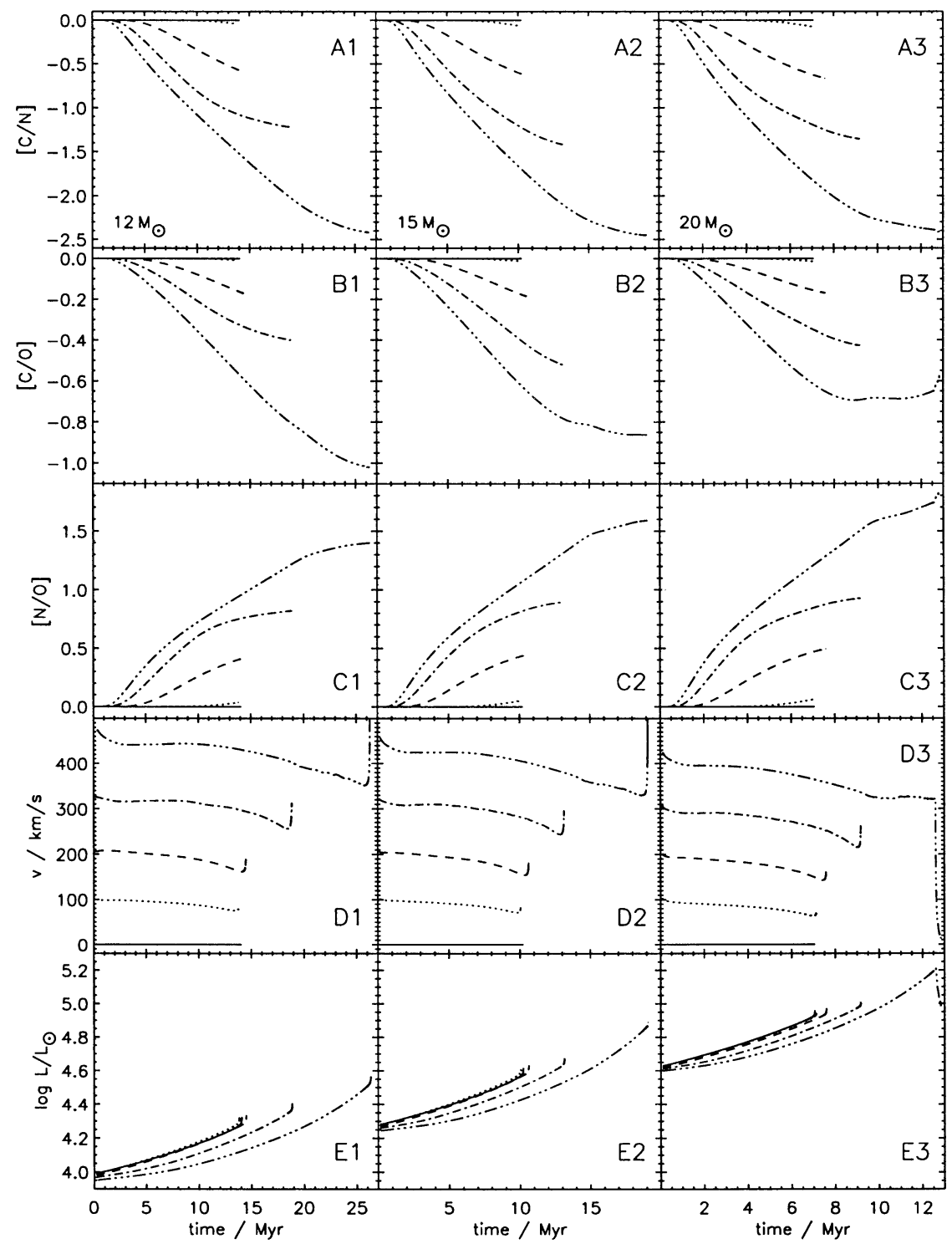

Figure 2. Evolution of surface properties of stellar models with rotation for initial masses of $12 \mathrm{M}_{\odot}$ (left panel), $15 \mathrm{M}_{\odot}$ (middle panel), and $20 \mathrm{M}_{\odot}$ (right panel) and various initial rotation rates (no rotation: solid lines, about 100, 200, 300, $450 \mathrm{~km} \mathrm{~s}^{-1}$ : dotted, dashed, dashdotted and dash-triple-dotted lines, respectively) as function of time throughout core hydrogen burning. Shown are the the logarithmic increment of surface abundance ratios with respect to the solar ratio, the equatorial rotational velocity, and the stellar luminosity (see Heger 1998, and Heger et al. 1999 for more details). 


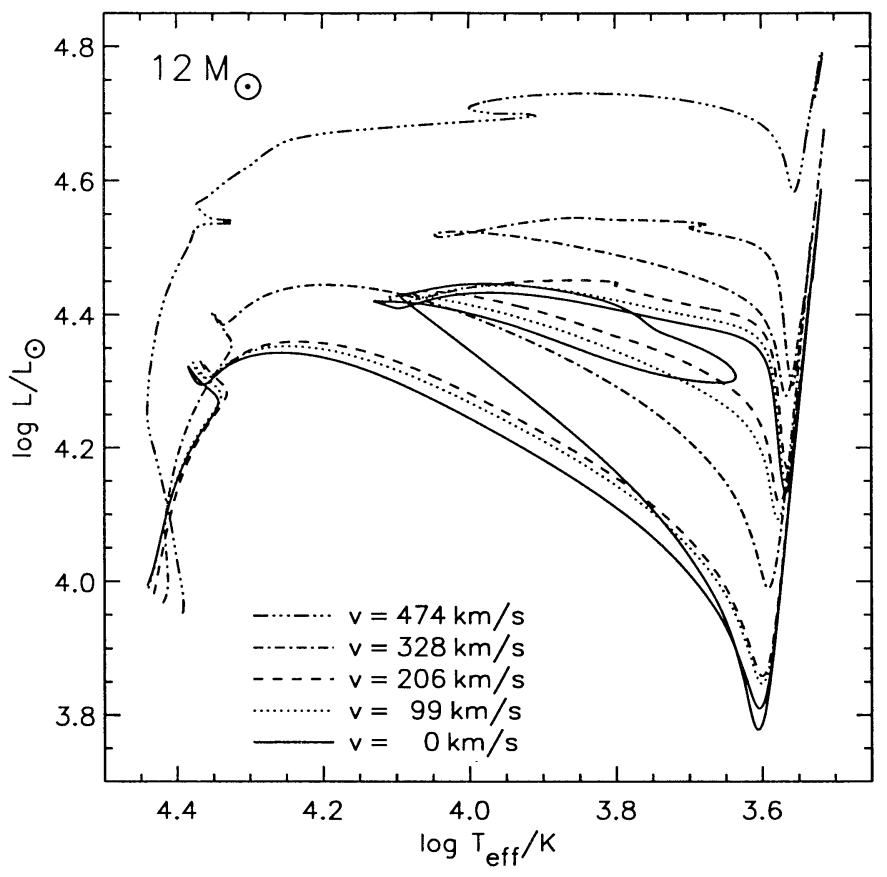

Figure 3. Evolutionary tracks in the HR diagram from the zero age main sequence to the pre-supernova position, for $12 \mathrm{M}_{\odot}$ stars with initial equatorial rotational velocities of $0 \mathrm{~km} \mathrm{~s}^{-1}, 99 \mathrm{~km} \mathrm{~s}^{-1}$ (dotted line), $206 \mathrm{~km} \mathrm{~s}^{-1}$ (dashed line), $328 \mathrm{~km} \mathrm{~s}^{-1}$ (dash-dotted line), and $474 \mathrm{~km} \mathrm{~s}^{-1}$ (dash-triple-dotted line). See Heger (1998) and Heger et al. (1999) for more details.

Rotation also increases the stellar mass loss rate (Langer 1998), which appears to be required by observations (Meynet et al. 1994). The anisotropy of the wind of rotating massive stars (Bjorkman \& Cassinelli 1993) may also be related to the $\mathrm{B}[\mathrm{e}]$ phenomenon (Langer \& Heger 1998), to the bipolarity of LBV nebulae (Langer et al. 1999a), and perhaps to the rings around SN 1987A (Heger \& Langer 1998).

In summary, rotation has the potential to assess most of the problems mentioned in Section 1 (see also Talon et al. 1997; Maeder 1999). It certainly needs to be considered in the context of star formation, which is the process determining the initial angular momentum in a star (possibly in relation with problem 6; Figer et al. 1998) and its distribution function. Moreover, in addition to the effects outlined here, rotation has further implications for nucleosynthesis (Langer et al. 1999b), supernova explosions, pulsar rotation (Langer et al. 1999c; Heger et al. 1999), and even $\gamma$-ray bursters (MacFadyen \& Woosley 1999)

Acknowledgments. The authors are very grateful to André Maeder, Stan Owocki, Regina Schulte-Ladbeck, Kim Venn and Stan Woosley for enlightening discussions. This work has been supported by the Deutsche Forschungsgemeinschaft through grants No. La 587/15-1 and La 587/16-1. 


\section{References}

Arnett, W.D., Bahcall, J.N., Kirshner, R.P., \& Woosley, S.E. 1989, ARA\&A, 27,629

Bjorkman, J.E., \& Cassinelli, J.P. 1993, ApJ, 409, 429

Blaha, C., \& Humphreys, R.M. 1989, AJ, 98, 1598

Braun, H., \& Langer, N. 1999, in preparation for A\&A

Crowther, P.A., \& Bohannan, B. 1997, A\&A, 317, 532

Emden, V.R. 1907, Gaskugeln, Leipzig

Figer, D.F., Najarro, F., Morris, M., McLean, I.S., Geballe, T.R., Ghez, A.M., \& Langer, N. 1998, ApJ, 506, 384

Fitzpatrick, E.L., \& Garmany, C.D. 1990, ApJ, 363, 119

Gummersbach, C.A., Zickgraf, F.-J., \& Wolf, B. 1995, A\&A, 302, 409

Heger, A. 1998, PhD Thesis, TU München

Heger, A., \& Langer, N. 1998, A\&A, 334, 210

Heger, A., Langer, N., \& Woosley, S.E. 1999, ApJ, in preparation

Howarth, I.D., Siebert, K.W., Hussain, G.A.J., \& Prinja, R.K. 1997, MNRAS, 284,265

Iben, I. Jr. 1974, ARA\&A, 12, 215

Kudritzki, R.P., Lennon, D.J., Haser, S.M., Puls, J., Pauldrach, A., Venn, K., \& Voels, S.A. 1996, in Science with the HST, STScI, P. Benvenuti et al., eds.

Langer, N. 1991, A\&A, 252, 669

Langer, N. 1992, A\&A, 265, L17

Langer, N. 1994, in Proc. $34^{\text {th }}$ Herstmonceux Conference on Circumstellar Media in the Late Stages of Stellar Evolution, R. Clegg et al., ed., Cambridge University Press, p. 1

Langer, N. 1998, A\&A, 329, 551

Langer, N., \& Maeder, A. 1995, A\&A, 295, 685

Langer, N., \& Heger, A. 1998, in proc. Workshop on B[e] stars, Paris 1997, C. Jaschek, A. M. Hubert, (eds.), Kluwer, 235

Langer, N., El Eid, M.F., \& Baraffe, I. 1989, A\&A, 224, L17

Langer, N., Heger, A., \& García-Segura, G. 1998, RvMA, 11, 57

Langer, N., García-Segura, G., \& Mac Low, M.-M. 1999a, ApJ, submitted

Langer, N., Heger, A., Woosley, S.E., \& Herwig, F. 1999b, in Nuclei in the Cosmos V, N. Prantzos, ed., Édition Frontières, Paris, in press

Langer, N., Heger, A., \& Woosley, S.E. 1999c, in proc. IAU-Symp. 193 on WolfRayet Phenomena in Massive Stars and Starburst Galaxies, K.A. van der Hucht et al., eds., ASP Conf. Ser., San Francisco, in press

MacFadyen A., \& Woosley, S.E. 1999, ApJ, submitted

Maeder A. 1999, in proc. IAU-Symp. 193 on Wolf-Rayet Phenomena in Massive Stars and Starburst Galaxies, K.A. van der Hucht et al., eds., ASP Conf. Ser., San Francisco, in press 
Meynet, G., Maeder, A., Schaller, G., Schaerer, D., \& Charbonnel, C. 1994, A\&AS, 103, 97

Pasquali, A., Langer, N., Schmutz, W., Leitherer, C., Nota, A., Hubeny, I., Moffat, A.F.J., Drissen, L., \& Robert, C. 1997, ApJ, 478, 340

Talon, S., Zahn, J.P., Maeder, A., \& Meynet, G. 1997, A\&A, 322, 209

Weaver, T.A., Zimmerman, G.B., \& Woosley, S.E. 1978, ApJ, 225, 1021

Woosley, S.E., Heger, A., Weaver, T.A., \& Langer, N. 1999, in SN 1987A: Ten years after, Eds. M.M. Phillips, N.B. Suntzeff, PASP, in press

\section{Discussion}

Nolan Walborn: In answer to a question during the talk, the two O3 stars on your $200 \mathrm{M}_{\odot}$ track are in associations. Sk $-67^{\circ} 211=\mathrm{HDE} 269810$ is next to a small HII region, and $\mathrm{Mk} 42$ is in the 30 Dor central cluster.

Paul Crowther has found an exact spectroscopic counterpart of the LMC Ofpe/WN9 stars in the Galaxy; it has an obscure name like NS4 or some such. He has classified subgroups of this class as WN9-11 and, with Bruce Bohannan, has proposed that the galactic OIafpe stars like (and including) HD 152408 should also be classified as WN9. So there are galactic counterparts of this LMC class.

George Sonneborn: If rotation-induced mass loss can produce the rings around SN1987A, does this also place the star in the right part of the HR diagram for a single-star explosion? Does this argue for or against binary or single-star models for the 1987A progenitor?

Langer: The post-red supergiant spin-up mechanism found by Heger \& Langer (1998) may certainly be relevant for shaping the inner ring; its effect on the outer rings still needs to be investigated. Since the spin-up occurs for all kinds of evolution from the Hayashi-line to hotter surface temperatures, it can not be used to discriminate between single star progenitor models and models invoking binary effects.

Philip Massey: Three comments, all concerning incompleteness:

(a) The "problem" with the main-sequence widening described by Fitzpatrick \& Garmany (1990 Ap.J., 363, 119) was really dominated by the incompleteness at the hot end - not the over abundance of stars past the MS. In other words, if you expect only a tiny fraction of MS stars to be seen past the "gap" you can believe there's a problem if you greatly underestimate the number of MS stars. With more complete data, and well determined incompleteness corrections (down to $25 \mathrm{M}_{\odot}$ anyway), there doesn't seem to be any problem in the number of stars across the HRD, at least using the Geneva (Maeder's) tracks using convection overshooting (Massey et al. 1995, ApJ, 438, 188).

(b) Neither the number of blue supergiants nor the number of red supergiants are known to an order of magnitude in the Milky Way or MC's - so does the ratio really mean anything? In a recent survey for red supergiants and WR stars in NGC 6822, M31, and M33 we do see an excellent correlation in number ratio (RSG/WR) with metallicity - so maybe this is no longer a problem to solve. 
(c) Finally let me note that there is one WN9/Ofpe star known in even the higher-than-solar metallicity galaxy M31; the Wolf-Rayet star OB69-WR2. Their emission is weak in terms of equivalent widths so they are hard to detect! See discussion in Massey \& Johnson (1998, ApJ, 505, 793).

Langer: (a) A post-main sequence gap is predicted by all massive star models, also by the Geneva models, and it can not coincide with the observed gap between the Fitzpatrick \& Garmany ledge and the red supergiant branch. Observations and theory are clearly in conflict here.

(b) The absolute numbers of blue or red supergiants are surely very uncertain. However, the relative numbers, and thus the blue/red supergiant number ratios at different metallicities, can conclusively be compared with each other (e.g., Langer \& Maeder 1995, A\&A, 295, 685).

Sally Oey: Your evolutionary tracks including rotation evolve at higher L. Won't this affect the evolutionary time scales and stellar winds?

Langer: Yes, it does. The life times get longer despite the increased luminosities, since the rotational mixing brings more "fuel" to the center. And the mass loss rates are considerably increased, not only due to the higher luminosities, but also due to the centrifugal force (Langer 1998, A\&A, 329, 551). 